\title{
The Functional Measurement Experiment Builder suite: Two Java-based programs to generate and run functional measurement experiments
}

\author{
Olivier Mairesse, Joeri Hofmans, and Peter Theuns \\ Vrije Universiteit Brussel, Brussels, Belgium
}

\begin{abstract}
We propose a free, easy-to-use computer program that does not requires prior knowledge of computer programming to generate and run experiments using textual or pictorial stimuli. Although the FM Experiment Builder suite was initially programmed for building and conducting FM experiments, it can also be applied for non-FM experiments that necessitate randomized, single, or multifactorial designs. The program is highly configurable, allowing multilingual use and a wide range of different response formats. The outputs of the experiments are Microsoft Excel compatible .xls files that allow easy copy-paste of the results into Weiss's FM CalSTAT program (2006) or any other statistical package. Its Java-based structure is compatible with both Windows and Macintosh operating systems, and its compactness $(<1 \mathrm{MB})$ makes it easily distributable over the Internet.
\end{abstract}

How does one judge a person's attractiveness as a function of information about this person's look and personality? Or how does information about one's amount of sleep during the previous night combine with information about the time of day when judging a person's degree of sleepiness? These issues can be studied using Anderson's functional measurement (FM) paradigm (Anderson, 1981). The main idea of FM is embedded within the theoretical framework of information integration, in which logic is illustrated by the integration diagram shown in Figure 1 (Anderson, 1981). In this integration diagram, three functions convert a set of stimuli into a single response. The valuation function follows the psychophysical law - that is, the process in which observable (physical) stimuli $S_{1}$, $S_{2}$, and $S_{3}$ engender concurrent psychological representations $s_{1}, s_{2}$, and $s_{3}$. Through psychological integration (the integration function), $s_{1}, s_{2}$, and $s_{3}$ are combined into a single implicit response $r$ and, subsequently, an observable response $R$ is generated by means of the response function. The logic of FM is that the integration function can be described by simple algebraic rules such as addition, averaging, and multiplication (for a more thorough review, see Anderson, 1981, 1982, 1996).

FM experiments allow for the simultaneous validation of two premises: (1) the algebraic rule that describes the psychological integration, and (2) the linearity of the response function (Anderson, 1981). Assuming that the second premise is true - that is, the response function is linear-different integration rules predict specific patterns in the factorial plot. For example, an additive inte- gration rule predicts a pattern of parallelism, whereas a multiplicative integration rule yields a linear fan pattern (Anderson, 1981, 1982). This means that perceiving one of the predicted patterns in the experimental data simultaneously supports the corresponding integration rule as well as the linearity of the response function. Therefore, this technique can be of interest for anyone who wants to study the integration of various factors affecting the perception of a specific construct, as well as for someone who wants to test a particular response scale regarding linearity (see Hofmans \& Theuns, in press; Hofmans, Theuns, \& Mairesse, 2007).

Carrying out FM experiments requires multifactorial designs in which compounded stimuli are to be evaluated in terms of perceived intensity on a response scale. Previous studies have - among other things - examined the perception of areas from weight and length information (Anderson \& Cuneo, 1978; Mullet \& Miroux, 1996; Mullet \& Paques, 1991), pain judgments from facial features information (de Sá Teixeira \& Oliveira, 2007) and judgments of sleepiness from information about homeostatic sleep pressure and the circadian phase (Mairesse, Hofmans, De Valck, Cluydts, \& Theuns, 2007). They have also investigated psychological constructs, like fear of contagion from unnamed diseases with different degrees of transmissibility and severity of prognoses (Rundall \& Weiss, 1994), as well as the willingness to forgive on the basis of information on intent, proximity of the relationship, given apologies, and the severity of consequences of the act (Girard, Mullet, \& Callahan, 2002). As this list of 


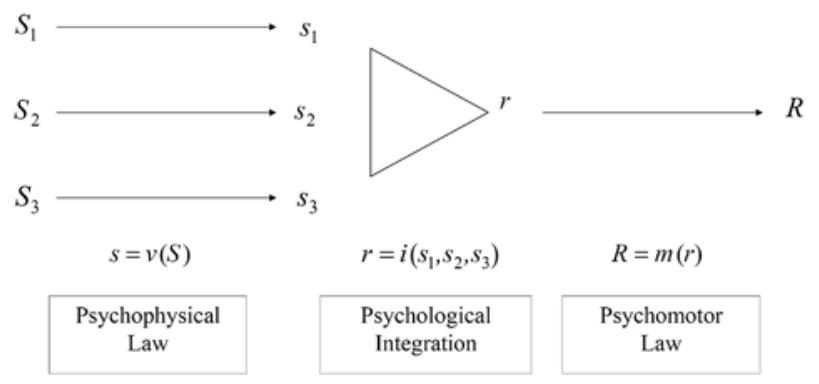

Figure 1. Functional measurement diagram (Anderson, 1981; Weiss, 2006): $S_{n}$, observable (physical) stimuli; $s_{n}$, the subjective stimuli; $r$, the subjective response; $R$, the observable response; $v$, valuation function; $i$, integration function; $m$, response function.

topics illustrates, the stimuli-as well as the number of factors in FM experiments - can be very diverse. Running FM experiments often require complicated factorial designs, full randomization procedures, and different types of response scales. Setting up such computer experiments often necessitates programming skills or the acquisition of an expensive experiment generator package. We propose the FM experiment builder suite as a free-of-charge, easyto-use experiment generator-runner requiring no prior knowledge of computer programming from the investigator. The FM experiment builder suite allows the inclusion of almost any text- or image-based stimulus and offers a wide range of different response formats such as category scales, visual analog scales and textual or numerical input. Its Java-based structure is compatible with both Windows and Macintosh operating systems, and its compactness $(<1 \mathrm{MB})$ makes it easily distributable over the Internet.

The procedure to be followed in order to create an FM experiment will be described using an example of an experiment on judgments of subjective sleepiness (Mairesse et al., 2007). In this study, participants had to estimate how sleepy they would feel after having slept a certain amount of time at a specific moment during the day. The stimuli were arranged according to a $2 \times 3$ full factorial design, with three repetitions. Factor 1 (prior sleep) consisted of two levels: $8 \mathrm{~h}$ and $2.5 \mathrm{~h}$ of sleep. Factor 2 represented three different moments across the day: 9 a.m., 11 a.m., and 1 p.m. Judgments were made using a visual analog scale labeled from very alert to very sleepy.

\section{FM WRITER}

The FM WRITER program is used to create the experiment. In order to run FM WRITER, two executable .jar files are needed: FM WRITER.jar and jxl.jar. The first opens the interface in which the experiments will be constructed; the latter operates as a cache in which all variables are stored during the production of the experiment. A screen capture of the main screen of the FM WRITER is displayed in Figure 2.

Once FM WRITER is opened, a number of text fields are activated to allow input of the project's name, the main instructions, the welcome text, and the final text, which is displayed after completion of the experiment. Checkboxes allow for the selection of sociodemographic input fields that can be incorporated in the FM experiment. The user can choose to build a first name-surname input field as well as input fields for the date of birth, the address, and the educational level of the participant. The input from the participant is used in order to create a participant-specific code for the participants' output file based on current clock time and name initials. If none of the sociodemographic input fields are selected, then the program generates a code based on the computer's clock time.

In order to get the participants acquainted with the experimental task, FM builder allows the user to incorporate practice trials, which are randomly sampled from the total stimulus set. The user determines the number of practice trials and is able to include a second screen of instructions after the trial run.

In most FM experiments, all factor $\times$ level combinations are repeatedly administered in order to minimize

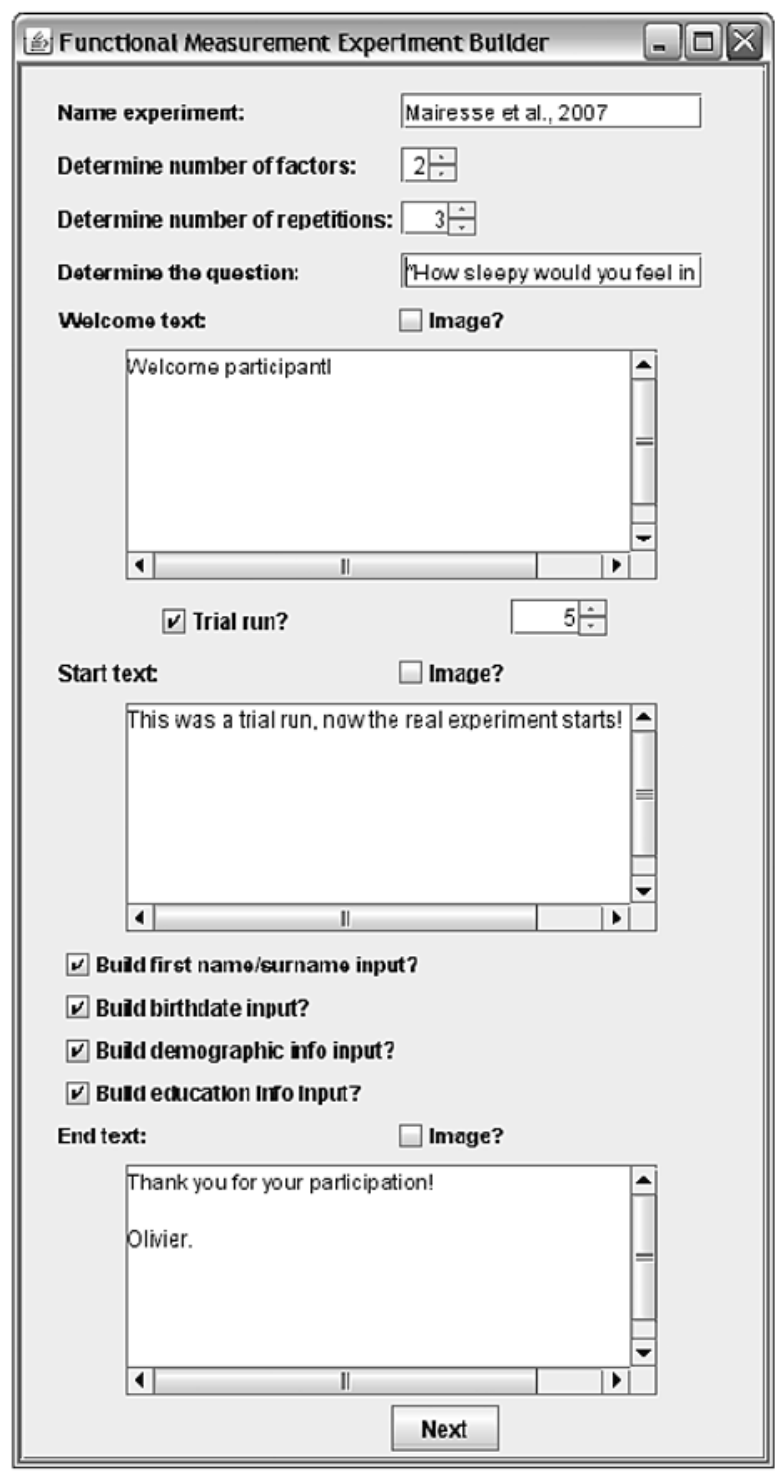

Figure 2. FM WRITER: Main input screen. 


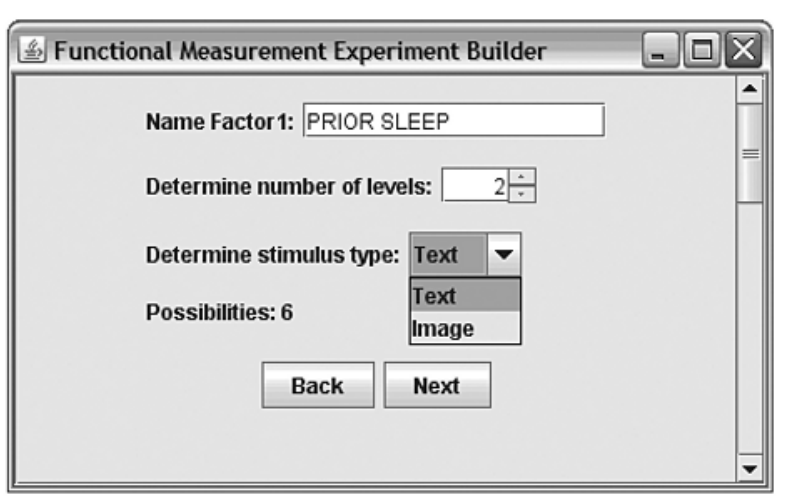

Figure 3. FM WRITER: Factor input screen.

variability-a procedure called a single-subject design (Anderson, 2002). Using combo boxes, the user can determine the number of stimulus repetitions as well as the number of factors in the experiment. In this first screen, the user can also formulate the question that will be presented on every trial - that is, How sleepy would you feel in the following situation? Clicking on the "Next" button calls the factor input screen (Figure 3).

Depending on the number of factors in the experiment, a number of subsequent screens are displayed one at a time. The user can determine the name of every factor (i.e., Factor 1: prior sleep) and also the number of levels for each factor (Factor 1: two levels). Moreover, the user can also determine whether the stimulus will be presented as text or as an image for each factor separately. When the experiment requires different modalities (i.e., text and images) for different levels of one factor, one can select the image type for this factor and create images displaying text for the textual levels. A progress indicator giving feedback of the number of combinations completed is displayed in order to keep track of the input process. In order to broaden the range of the responses of the participant or to hide the factorial structure of the experiment, filler (distractor) items can be included in the design. Doing this can be easily achieved by using text saved as image. Then, instead of specifying a main question in the FM Builder program, the user may leave a blank space and include the item in the image stimulus. To preserve the factorial structure of the output, the user simply defines a single factor and imports the image stimuli according to the desired factorial design (i.e., Factor 1-Level 1; Factor 1-Level 2, etc.). Using images is also useful when entire paragraphs are used as stimuli (see Girard et al., 2002).

Clicking on the "next" button calls the level input screen for text or image input. At this moment, the stimuli can be inserted by the user (i.e., Factor 1-Level 1: You slept eight hours). The input procedure for textual stimuli is similar to the one of the previous screen. For every factor, the name of the factor is displayed, once again in order to keep track of the whole procedure. In some FM experiments, a "blank" level must be incorporated in the design in order to distinguish the additive from the averaging model (see
Anderson, 1981, 1982, 1996). The inclusion of a "blank" level can be achieved by simply inserting a space in the text field and validating it by clicking on "Enter."

For pictorial stimuli, the program allows browsing through the users' documents. When an image is chosen, the caption of the level indicates the location of the file. At this point, the FM Builder suite supports .jpg and .gif image formats. When using pictorial stimuli, a folder for every factor is created in which all corresponding images are stored. In order to run the experiment properly later on, all folders containing the images must be present.

Next is the scale-builder screen. The scale builder offers the possibility to build an extensive variety of response scales such as category scales, visual analog scales, and text or number input (i.e., numerical estimation). Three types of scales can be selected from the drop-down menu: "Category," "Slider," and "Text." "Slider" refers to visual analogue scales, "Category" to category scales, and "Text" to textual or numerical input. Each scale type is presented horizontally by default and can be presented vertically by validating the appropriate checkbox. Another checkbox ("Label scales with images?") offers the opportunity to insert an image for the response scale, an image for every category for a category scale, and a single image for a slider. In order to avoid participants clicking through the experiment, the user can determine the delay time (i.e., $2 \mathrm{sec}$ ) after which the "Next" button appears.

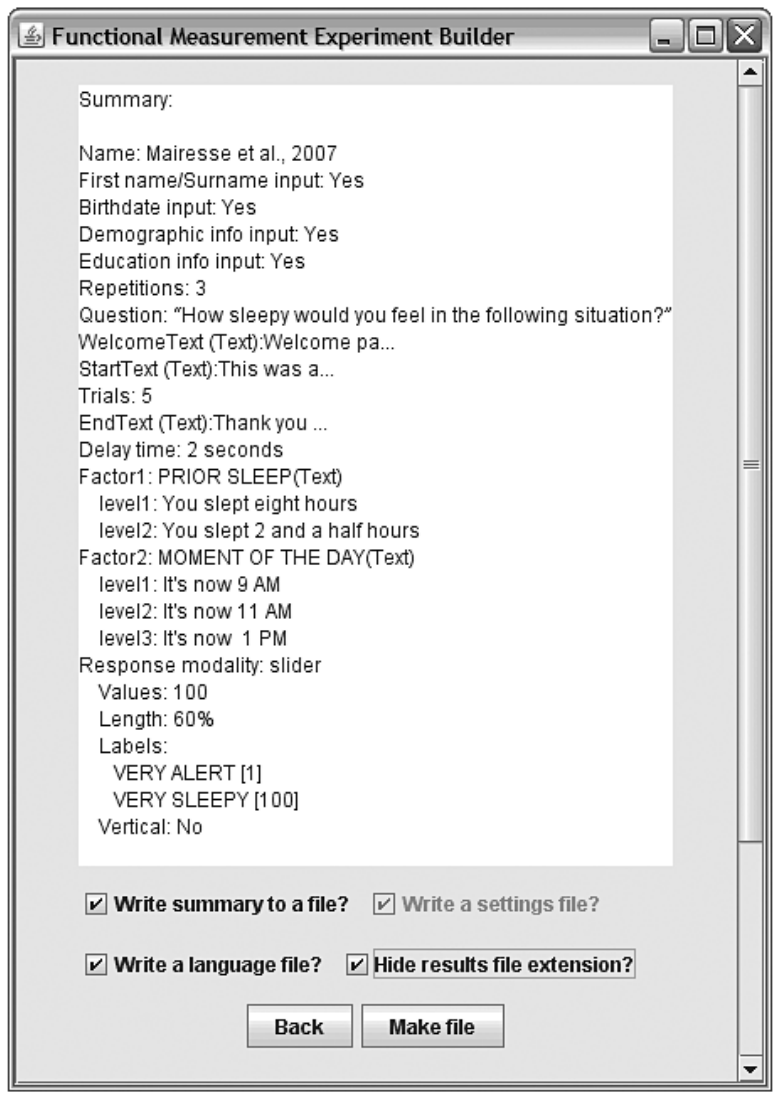

Figure 4. FM WRITER: Summary screen. 


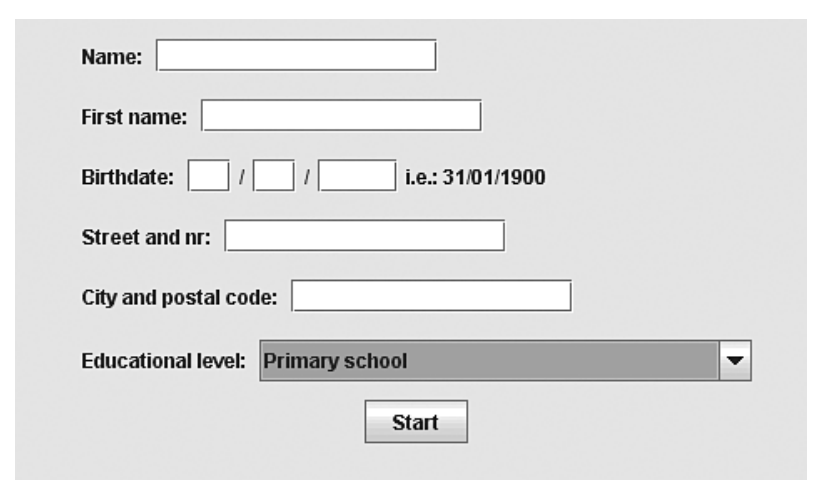

Figure 5. FM READER: Sociodemographic input screen.

Since it is possible to build a large number of different response scales through the combination of images, verbal information, sliders, categories, and text fields, a brief overview of the three types of scale that can be built will be given below.

1. When either the "Slider" or the "Category" option is chosen from the drop-down box, clicking on the "Next" button calls up the scale-tweaking screen. Building a regular visual analog scale can be done by choosing two categories to correspond with the end anchors of the scale (i.e., very alert and very sleepy) and setting " 100 " as the number of values of the slider. Increasing the number of categories allows the user to build label-anchored visual analog scales, since each value or position on the slider can be associated with a label. In the current version of the FM Builder suite, a maximum of 10 labels is allowed because of layout purposes. The width of the slider can be manipulated in terms of the percentage of the width of the screen. This means that the slider will take the same proportion of the screen width on every monitor.

2 . When the option to build a category scale is chosen in the previous screen, all previous tweaking options are disabled, and only the "number of radio buttons" option is enabled. This version of FM WRITER supports a maximum of 20 radio buttons. For category scales, the next screen offers the possibility to define labels. A similar screen is prompted when the option "label scales with images" has been selected in the scale-builder screen except that instead of entering text, the user can browse through his or her documents and assign different images to any of the available scale positions. All images are resized in order to fit the screen resolution of the computer that will be used to run FM READER. When the user enables the vertical display option, the images will be displayed next to the slider in the FM READER.

3. When the option "text" is selected in the scale-builder screen, the instruction input screen of the FM WRITER is displayed. Using the text box, the user can easily write an appropriate instruction, and FM READER will display this instruction and the text input field below the stimuli.

After completion of the scale building procedure, the final FM WRITER screen is displayed (Figure 4). This screen shows a summary of the operations undertaken in the building procedure. By clicking the "Make file" com- mand, an .exp file with default layout settings is created. This file contains the experiment and can be read only by the FM READER.

The final screen possesses some interesting features for the user. The summary displayed on the final screen can be saved in a text document in order to reconstruct the experiment. The user may also create a language file with the purpose of translating a few lines of text regarding the input of sociodemographic variables. Moreover, a display settings file can be created in order to fine tune the layout of the experiment.

The default output file of the experimental results is a Microsoft Excel-compatible .xls file. In order to avoid easy recognition of the output file by the participant, the file extension can be modified into an .fmb file, which cannot be opened in Excel by simply double clicking it. Renaming the output file into .xls or .csv reenables the Microsoft Excel compatibility at once. This option can be reset to false in the settings file. Further customization of the layout of the experiment will be discussed in the next section.

\section{FM READER}

This subprogram runs the experiment created with FM WRITER. When an .exp file has been created, the user can access the experiment by double clicking on the FM READER.jar. When clicking on the "Next" button after the welcome screen, the sociodemographic input screen appears (Figure 5).

After the sociodemographic input screen, the actual experiment begins. The READER program presents stimuli randomized within repetitions. That is, depending on the number of repetitions specified by the user in the WRITER program, FM READER will present all factor $\times$ level stimulus combinations in random order a first time and then initiate a new randomization of the stimuli until all repetitions are presented. Figure 6 displays a view from the FM experiment on sleepiness judgments we used as an example.

Altering the layout of the experiment is an interesting feature of the FM Builder suite. The language used for the recording of sociodemographics can be changed by translating the items in the oplniv.txt file. ${ }^{1}$ English text can be replaced easily by overwriting it in another language. Variable names must remain unaltered in order to maintain the functionality of the software. Saving the file with the translated text is sufficient for the translation to be effective in the FM READER, as long as the program is not yet open. When FM READER is already open, restarting the software is required for the changes to take effect.

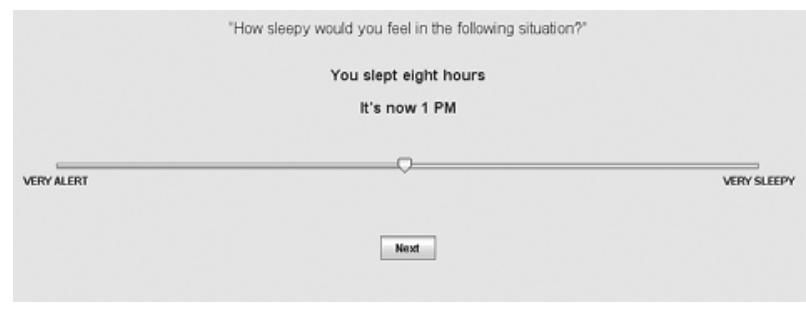

Figure 6. FM READER: Experiment view. 
Table 1

Example of a Windows Excel Output Generated by the FM READER

\begin{tabular}{ccllr}
\hline Participant Code & Repetition & \multicolumn{1}{c}{ Factor 1 } & \multicolumn{1}{c}{ Factor 2 } & Results \\
\hline 1475458QP & 1 & You slept eight hours & It's now 9 AM & 51 \\
1475458QP & 1 & You slept 2 and a half hours & It's now 9 AM & 42 \\
1475458QP & 1 & You slept eight hours & It's now 11 AM & 65 \\
1475458QP & 1 & You slept 2 and a half hours & It's now 11 AM & 47 \\
1475458QP & 1 & You slept eight hours & It's now 1 PM & 70 \\
1475458QP & 1 & You slept 2 and a half hours & It's now 1 PM & 20 \\
1475458QP & 2 & You slept eight hours & It's now 9 AM & 100 \\
1475458QP & 2 & You slept 2 and a half hours & It's now 9 AM & 55 \\
1475458QP & 2 & You slept eight hours & It's now 11 AM & 38 \\
1475458QP & 2 & You slept 2 and a half hours & It's now 11 AM & 55 \\
1475458QP & 2 & You slept eight hours & It's now 1 PM & 72 \\
1475458QP & 2 & You slept 2 and a half hours & It's now 1 PM & 47 \\
1475458QP & 3 & You slept eight hours & It's now 9 AM & 75 \\
1475458QP & 3 & You slept 2 and a half hours & It's now 9 AM & 37 \\
1475458QP & 3 & You slept eight hours & It's now 11 AM & 68 \\
1475458QP & 3 & You slept 2 and a half hours & It's now 11 AM & 33 \\
1475458QP & 3 & You slept eight hours & It's now 1 PM & 47 \\
1475458QP & 3 & You slept 2 and a half hours & It's now 1 PM & 41 \\
\hline
\end{tabular}

Advanced tweaking of the layout can be completed by altering default values in the Settings_name experiment. txt file. An overview of the functions is provided in the instruction manual included with the software package.

In Table 1, an example of a Windows Excel output for 1 participant is displayed. The results can be copied and pasted in any spreadsheet. The results are saved as text; thus, converting them to numbers may be required for some programs. In order to structure a data file for multiple participants, all .xls results files can be merged using an .xls or a .csv file merger, or they can be copied and pasted one by one. These results are CalSTAT FM ready (Weiss, 2006).

\section{AUTHOR NOTE}

O.M. is supported by Grant OZR1023BOF of the Vrije Universiteit Brussel. J.H. is supported by Grant OZR1041BOF of the Vrije Universiteit Brussel. We are especially grateful to Wim Rongé for the art of coding. A free copy of the FM Experiment Builder suite is available by contacting O.M. Address correspondence to O. Mairesse, Vrije Universiteit Brussel, Faculty of Economic, Social and Political Sciences and Solvay Business School, Pleinlaan 2, B-1050 Brussels, Belgium (e-mail: olivier.mairesse@vub.ac.be).

\section{REFERENCES}

ANDERSON, N. H. (1981). Foundations of information integration theory. New York: Academic Press.

ANDERSON, N. H. (1982). Methods of information integration theory. New York: Academic Press.

ANderson, N. H. (1996). A functional theory of cognition. Mahwah, NJ: Erlbaum.

Anderson, N. H. (2002). Methodology and statistics in single-subject experiments. In J. Wixted \& H. Pashler (Eds.), Stevens' Handbook of experimental psychology: Vol. 4. Methodology in experimental psychology (pp. 301-337). New York: Wiley.

Anderson, N. H., \& Cuneo, D. O. (1978). The height + width rule in children's judgments of quantity. Journal of Experimental Psychology: General, 107, 335-378.

de SÁ Teixeira, N., \& Oliveira, A. (2007). Multidimensional quantitative semantics of pain: A nomothetic-idiographic approach through functional measurement. Teorie \& Modeli, XII(1-2), 155-166.

Girard, M., Mullet, E., \& Callahan, S. (2002). Mathematics of forgiveness. American Journal of Psychology, 115, 351-375.

Hofmans, J., \& Theuns, P. (in press). On the linearity of predefined and self-anchoring visual analog scales. British Journal of Mathematical \& Statistical Psychology.

Hofmans, J., Theuns, P., \& MaIresse, O. (2007). Impact of the number of response categories on linearity and sensitivity of "self-anchoring scales": A functional measurement approach. Methodology, 3, 160-169.

Mairesse, O., Hofmans, J., De Valck, E., Cluydts, R., \& Theuns, P. (2007). The linearity of subjective sleepiness measures as an additional support for the interaction of the circadian and homeostatic processes in sleepiness. Teorie \& Modeli, XII(1-2), 289-298.

Mullet, E., \& Miroux, R. (1996). Judgment of rectangular areas in children blind from birth. Cognitive Development, 11, 123-139.

Mullet, E., \& Paques, P. (1991). The height + width $=$ area of a rectangle rule in five-year-olds: Effects of stimulus distribution and graduation of the response scale. Journal of Experimental Child Psychology, 52, 336-343.

Rundall, C. S., \& Weiss, D. J. (1994). Nurses' fear of contagion: A functional-measurement analysis. Medical Decision Making, 14, 40-46.

WEIss, D. J. (2006). Analysis of variance and functional measurement: A practical guide. Oxford: Oxford University Press.

\section{NOTE}

1. Adaptations to the oplniv.txt file must be performed before compiling the experiment or the changes will have no effect. Changes in the language_name experiment.txt file can be performed without having to recompile the experiment.

(Manuscript received July 20, 2007; revision accepted for publication September 18, 2007.) 